\title{
LESÕES ANATOMOPATOLÓGICAS PRESENTES NA INFECÇÃO POR DIOCTOPHYMA RENALE (GOEZE, 1782) EM CÃES DOMÉSTICOS (CANIS FAMILIARIS, LINNAEUS, 1758)
}

\author{
(Anatomopathologic lesions found in Dioctophyma renale (Goeze, 1782) infections in \\ domestic dogs (Canis familiaris, LINNAEUS, 1758)
}

LEITE, L.C. ${ }^{1}$; CÍRIO, S.M. ${ }^{1}$; DINIZ, J.M.F. ${ }^{2}$; LUZ, E. ${ }^{3}$; NAVARRO-SILVA, M.A. ${ }^{3}$; SILVA, A.W.C. ${ }^{3}$; LEITE, S.C. ${ }^{4}$; ZADOROSNEI, A.C. ${ }^{4}$; MUSIAT, K.C. ${ }^{4}$; VERONESI, E.M. ${ }^{4}$; PEREIRA, C.C. ${ }^{4}$

\author{
${ }^{1}$ Professores - Pontifícia Universidade Católica do Paraná - PUC-PR tudolica@pop.com.br; \\ ${ }^{2}$ Professor Aposentado - Universidade Federal do Paraná - UFPR; \\ ${ }^{3}$ Professores - Universidade Federal do Paraná - UFPR; \\ ${ }^{4}$ Acadêmicas - Curso de Medicina Veterinária - PUC-PR ana_bazo@brturbo.com.br.
}

RESUMO - Os autores descrevem as lesões anatomopatológicas de onze casos de parasitismo por Dioctophyma renale (Goeze, 1782), na cidade de Curitiba - PR, Brasil, constatados em necrópsias e achados clínicos em 1960 cães, no período de 1979 a 2004.

Palavras-chave: Dioctophyma, parasita, lesões.

ABSTRACT - The authors describe the anatomopathologic lesions of eleven cases of parasitism by Dioctophyma renale (Goeze, 1782), in Curitiba (PR, Brazil), found among 1960 necropsies carried out between 1979 and 2004 in dogs.

Key-words: Dioctophyma, parasite, lesions.

\section{Introdução}

O nematóide parasita Dioctophyma renale (GOEZE, 1782) localiza-se preponderantemente no rim direito, ou livre na cavidade abdominal de seus hospedeiros, causando destruição progressiva das camadas cortical e medular, e reduzindo o órgão a uma cápsula fibrosa. No hospedeiro definitivo, as fêmeas adultas de $D$. renale podem alcançar tamanhos que variam entre quinze $\mathrm{cm}$. a um $\mathrm{m}$. de comprimento por 4 a $5 \mathrm{~mm}$. de diâmetro , e os machos adultos entre quinze a quarenta $\mathrm{cm}$. de tamanho por 5 a $10 \mathrm{~mm}$. de diâmetro.

De acordo com MAYRINK et al.(2000) o ciclo biológico do parasita tem como hospedeiros intermediários peixes de água doce, anelídeos aquáticos e rãs, que são ingeridos pelos hospedeiros definitivos, normalmente carnívoros domésticos e selvagens. No Brasil, o cão é o mais afetado dentre os animais domésticos (ALENCAR FILHO, 1966; AMATO et al. 1976) enquanto felinos, eqüinos, bovinos e suínos são parasitados com menor intensidade (DACORSO FILHO et al., 1954;
NEVES e MORAES, 1980). Em animais silvestres, foi observado parasitismo em quati (Nasua nasua, L.), furão (Galictis cuja), loboguará (Crysocyon brachiurus, Illiger) e preguiça (Choloepus didactylus, Linnaeus, 1785) (DACORSO FILHO et al. 1954; ROCHA e GRECHI, 1965; COSTA e FREITAS,1967; BARROS et al.,1990), ao passo que HANJAN et al. (1968); GUTIERREZ et al. (1989); URANO et al. (2001); IGNJATOVIC et al. (2003) registraram parasitismo em seres humanos.

A primeira descrição deste helminto no Brasil se deve a MOLIN (1860), em um lobo-guará (Crysocyon brachiurus, Illiger). Outros registros ocorreram nos Estados de Minas Gerais (COSTA e LIMA, 1988), São Paulo (PACHECO et al. 1965; SOUZA JÚNIOR e PADUA, 1977; TORRES et al., 2001), Pará (MIRANDA et al., 1992), Santa Catarina (NEVES et al.,1980), Rio Grande do Sul (SCHIMIDT, 1924; GONÇALVES et al. 1961; LARA et al, 1981; MARQUES et al., 1999), Rio de Janeiro (PROENÇA, 1935; DUARTE, 1981), Espírito Santo (BARROS, 1971) e Paraná (GIOVANNONI e MOLFI, 1960; FERNANDES, 1965; BARROS et al., 1990; 
BRUN et al. 2002). Recentemente, COSTA et al. (2004) descreveram um caso de dioctofimose em um canino de quatro anos de idade que albergava no rim direito uma fêmea de $D$. renale, medindo aproximadamente $54 \mathrm{~cm}$ de comprimento; estes autores concluíram que a dioctofimose ocorre preferencialmente em cães errantes, com hábitos alimentares pouco seletivos.

A predominância de $D$. renale no rim direito foi descrita por diversos pesquisadores brasileiros: PROENÇA (1935), DACORSO FILHO et al. (1954), GIOVANNONI e MOLFI (1960), MIGLIANO e MATERA (1966), NEVES et al. (1983), COSTA e LIMA (1988), MARQUES et al. (1999), PAES-DE-ALMEIDA e THOMÉ (1999), TORRES et al.(2001). GALVÃO et al. (1999) observaram a presença do parasita no rim esquerdo de um cão macho, com destruição maciça do cortex medular. NEVES et al. (1983) registraram três casos de helmintos livres na cavidade abdominal, além de achados em linfonodo mesentérico, cavidade torácica e entre lobos hepáticos. AUGUSTO FILHO et al. (1999) relataram achado de cinco parasitas (três machos e duas fêmeas) no rim esquerdo e sete (quatro machos e três fêmeas) no rim direito de uma cadela SRD com quatro anos de idade. Foi observada destruição total do parênquima e dilatação da pelve com áreas de perfurações. MONTEIRO et al. (2002) descreveram o encontro de trinta e quatro helmintos em um cão de dois anos eutanasiado e submetido à necropsia, sendo seis parasitas adultos no interior da cápsula renal direita, cujo parênquima encontrava-se totalmente destruído, e vinte e oito exemplares (dezoito adultos machos e fêmeas e dez formas imaturas) de $D$. renale na cavidade abdominal. Estudo retrospectivo realizado por DINIZ et al. (1996) descreveu lesões no parenquima renal, causadas por $D$. renale em Lobos-Guará jovens e adultos, mantidos em cativeiro no Zoológico de São Paulo, Brasil. Verificando as causas de mortalidade de animais cativos com base no registro de nascimento e óbito no International Studbook for Maned Wolf, no período de 19801998, MAIA et al. (2002) relataram óbitos em 7 animais capturados na natureza, decorrentes de infecção provacada por $D$. renale.

SADIGHIAN e AMINI (1967) no Irã, destacaram o encontro de 27 exemplares em apenas um cão, e GARGILI et al. (2002) na Turquia, registraram a presença de um parasita adulto de $D$. renale livre na cavidade abdominal de um cão.

O objetivo do presente trabalho é a descrição das lesões anatomopatológicas observadas em infecção de cães domésticos por $D$. renale, bem como o registro desse parasito em Curitiba, Paraná.

\section{Material e Método}

Foram estudados onze casos de dioctofimose em cães, a partir de material proveniente de 1546 necropsias realizadas no Hospital Veterinário da UFPR; dois registrados em clínicas veterinárias de Curitiba (Paraná), e 412 necropsias realizadas em aulas práticas de Anatomia Patológica Veterinária, da Pontifícia Universidade Católica do Paraná PUCPR no período entre 1979 a 2004. Todos os animais avaliados eram adultos, sem raça definida. Foram identificados e analisados sete exemplares fêmeas e quatro machos de $D$. renale, encontrados no rim direito e cavidade abdominal de onze cães. Sedimento urinário e líquido ascítico dos animais infectados foram coletados para análise. Os ovos encontrados foram medidos e fotografados. Um dos rins foi analisado macro e microscopicamente, fixandose seus fragmentos em solução de formol a $10 \%$, e mediante desidratação, diafanização e incluisão em parafina. Cortes histológicos de cinco $\mu \mathrm{m}$ de espessura, foram corados pelas técnicas de Hematoxilina de Harris e Eosina, Tricrômico de Mallory e Azul de Toluidina. Dois casos provenientes de clínicas veterinárias de Curitiba (PR) com suspeita clínica, foram confirmados por diagnóstico laboratorial, através de análise de sedimento urinário e de líquido oscístico, ambos contendo numerosos ovos de $D$. renale.

\section{Resultados e Discussão}

A presença de onze exemplares $(0,56 \%)$ de $D$. renale (FIGURA 1) (sete fêmeas e quatro machos) foi detectada em quatro cadelas e sete cães, de material procedente de 1960 animais sem raça definida avaliados. 
Lesões anatomopatológicas presentes na infecção por Dioctophyma renale (GOEZE, 1782) em cães domésticos...

Sete helmintos foram encontrados no rim direito e quatro na cavidade peritoneal. Esta freqüência é inferior à registrada em LagesSC por NEVES et al. (1983), que obtiveram treze animais positivos $(5,3 \%)$ em 244 necropsias.
Dos onze casos confirmados, oito resultaram de achados ocasionais em necropsias de cães levados a óbito por diferentes causas, dois tiveram diagnóstico confirmado através de exame de sedimento urinário e líquido ascítico, e um durante procedimento cirúrgico.

FIGURA 1 - EXEMPLAR ADULTO DE D. renale, EM CÃO ADULTO.

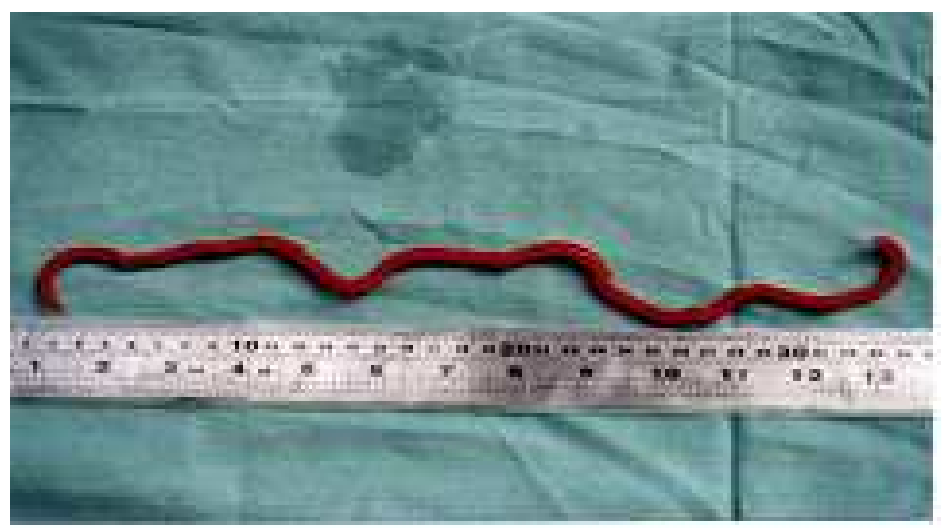

FIGURA 2 - EXEMPLAR DE D. renale PASITANDO O RIM DIREITO DE CÃO ADULTO.

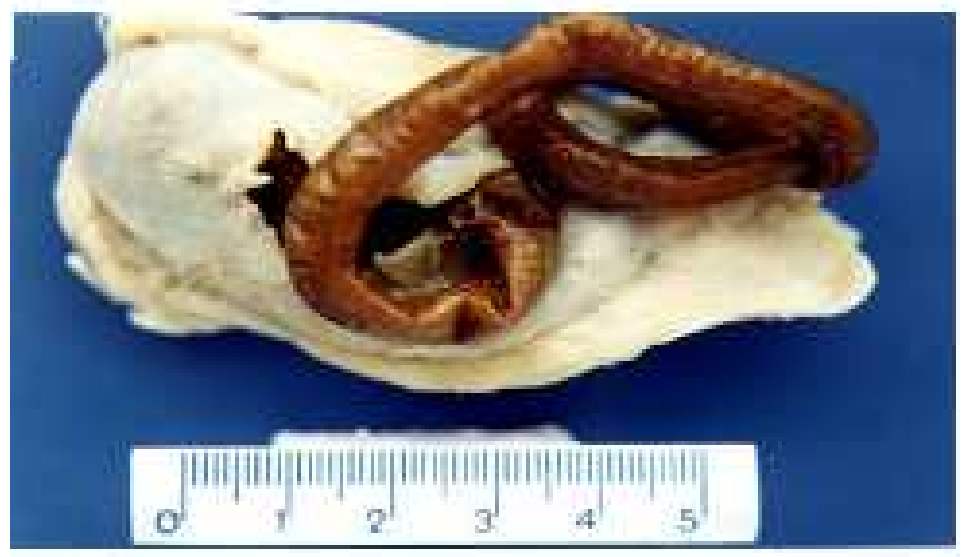

FIGURA 3 - OVO DE D.renale ENCONTRADO EM SEDIMENTO URINÁRIO DE CÃO ADULTO. OBJETIVA 40x.

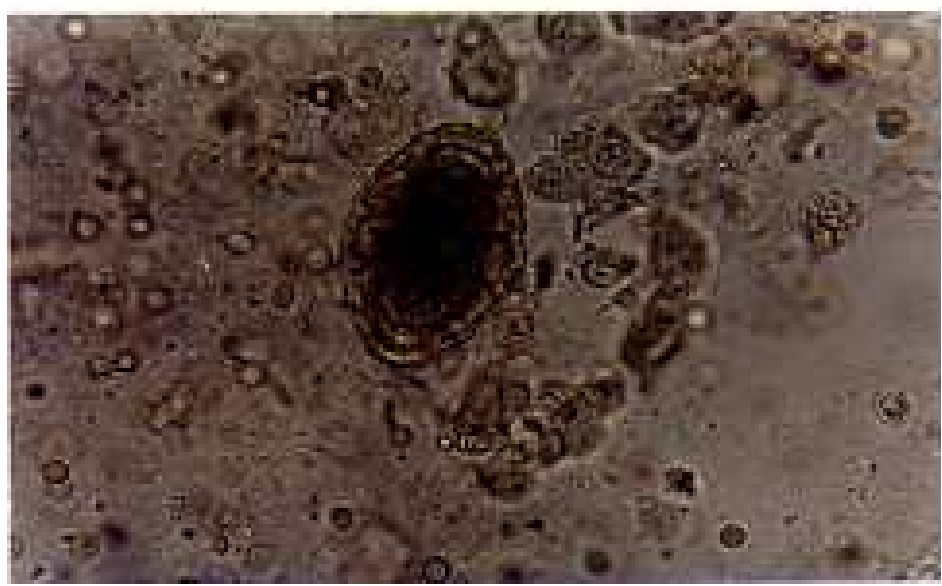


FIGURA 4 - REDUÇÃO DA CAMADA CORTICAL RENAL COM PROLIFERAÇÃO DE TECIDO CONJUNTIVO FIBROSO; TUBULOS CONTORNADOS DILATADOS; INTENSA INFILTRAÇÃO HISTIOLINFOPLASMOCITÁRIA NA CAMADA MEDULAR DE CÃO ADULTO, parasitado por $D$. renale. TRICROMICO DE MALLORY, OBJETIVA 10x.

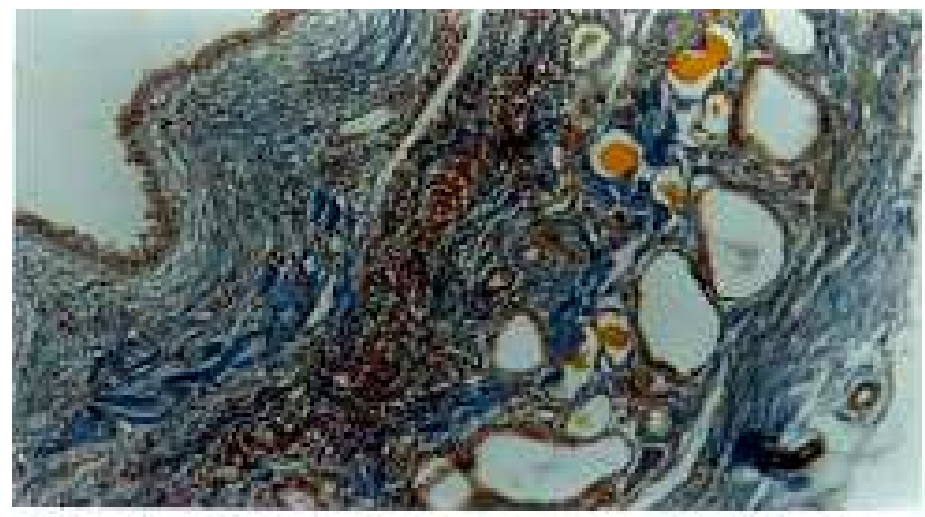

FIGURA 5 - CAMADA CORTICAL RENAL COM GLOMERULOS IRREGULARES EATROFIADOS; TUBULOS CONTORNADOS COM LUZ REDUZIDA E REVESTIDOS POR CÉLULAS APLAINADAS EM CÃO ADULTO, parasitado por $D$. renale.. HEMATOXILINA E EOSINA, OBJETIVA 40x.

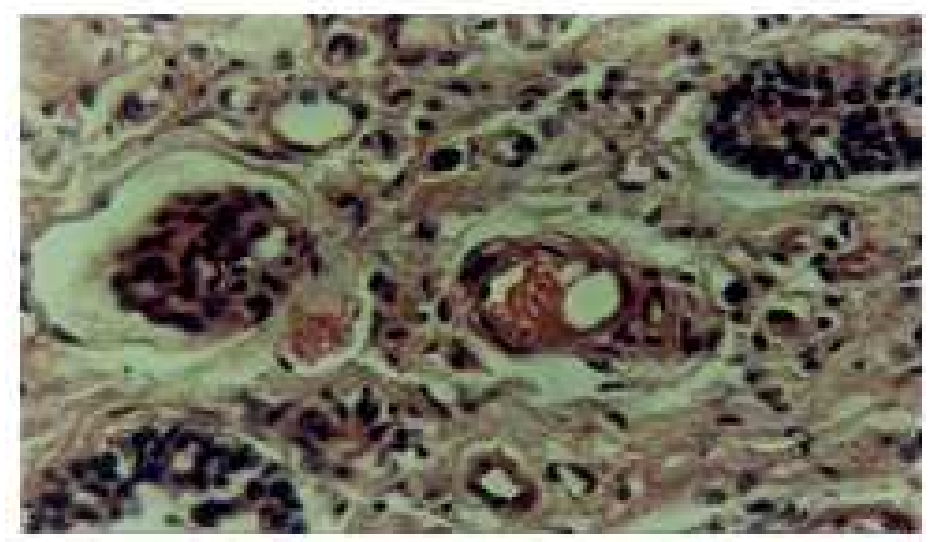

FIGURA 6 - EPITÉLIO DA PELVE RENAL COM HIPERPLASIAE DESCAMAÇÃO CELULAR EM CÃO ADULTO, PARASITADO POR D. renale. HEMATOXILINA EOSINA, OBJETIVA 40x.

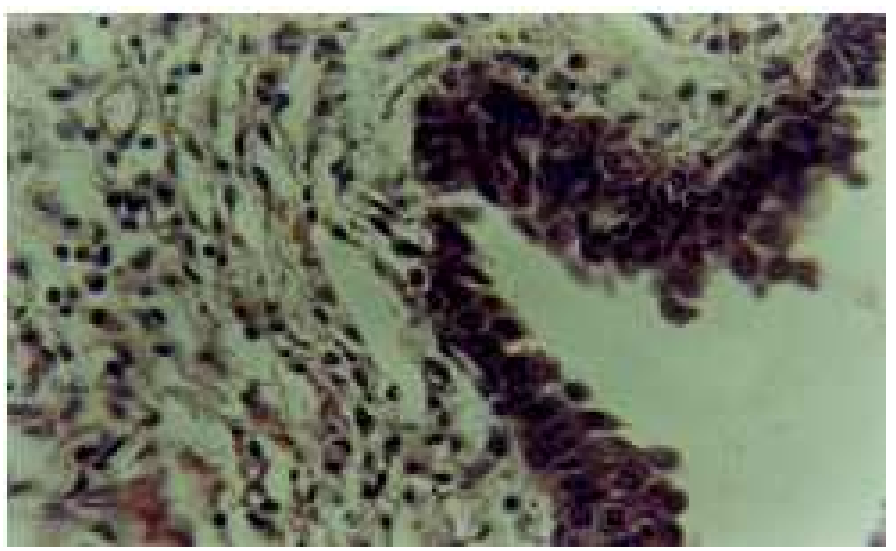


Lesões anatomopatológicas presentes na infecção por Dioctophyma renale (GOEZE, 1782) em cães domésticos...

Todos os animais infectados apresentavam hipertrofia compensatória do rim esquerdo, coincidindo com relatos de DACORSO FILHO et al. (1954), MIGLIANO e MATERA (1966), NEVES et al. (1983) e ACHA e SZYFRES (1986). O rim direito estava consistente à palpação, resistente ao corte com faca, com cápsula espessa e superfície da pelve bastante dilatada e irregular, formando grandes cavidades onde os helmintos estavam alojados (FIGURA 2). Foi observada atrofia por compressão do parênquima renal. No exame de sedimentos urinário (FIGURA 3) e ascítico, foram encontrados numerosos ovos, detalhe também observado por NEVES et al. (1983). Os cortes histológicos dos rins parasitados apresentavam espessamento da cápsula renal com neovascularização, hiperemia e infiltração histiolinfoplasmocitária, e acentuada redução da camada cortical a uma área delgada com intensa proliferação de tecido conjuntivo fibroso, fibras colágenas espessas e irregulares distribuídas em feixes paralelos, intensa infiltração intersticial histiolinfoplasmocitária e presença de alguns mastócitos. A luz tubular se achava reduzida, irregular e com células aplainadas. Outros túbulos se apresentaram dilatados, com necrose de células epiteliais (FIGURA 4), estruturas glomerulares raras, irregulares e atrofiadas (FIGURA 5). Presença de espessamento da cápsula parietal de Bowman e espaços de Bowman dilatados irregularmente, contendo em seu interior material amorfo, posteriormente corado por Eosina. Material idêntico também foi encontrado na luz tubular, coincidindo com achados registrados por NEVES et al. (1983). A camada medular apresentava-se totalmente fibrosa, infiltrada por histiócitos, linfócitos, plasmócitos e alguns mastócitos. Constatou-se neoformação vascular e vestígios de túbulos renais, alguns dilatados, com epitélio plano e evidências de hemorragias e necrose em algumas áreas, semelhante à descrição apresentada por MIGLIANO e MATERA (1966). O epitélio pelviano apresentou irregularidade, às vezes ausente ou hiperplásico, com descamação celular (FIGURA 6). Em algumas áreas, observouse a presença de células tumefatas, algumas vacuolizadas, com necrose e hemorragia.

\section{Conclusão}

Os dados obtidos nesse trabalho permitem concluir que:

- alterações histopatológicas foram representadas pela proliferação de tecido conjuntivo fibroso, dilatação tubular, infiltração histiolinfoplasmocitária, pronunciada atrofia glomerular além de hiperplasia e descamação celular acentuada do epitélio da pelve renal;

- os achados hitopatológicos traduzem os aspectos macrscópicos de atrofia de parênquima renal e distruição das camadas anatômicas que constituem o órgão.

\section{Agradecimentos}

Agradecemos às universidades (UFPR e PUC) pelo apoio e realização do presente trabalho, bem como a Clínica Veterinária Derosso Ltda. e ao Hospital Veterinário Clinivet Ltda. pelo material gentilmente fornecido.

Ao Prof. Eurides de Moura Leite, pela revisão ortográfica.

\section{REFERÊNCIAS}

ACHA, P.N.; SZYFRES, B. Dioctofimosis. In: ACHA, P.N.; SZYFRES, B. Zoonosis y Enfermedades Transmisibles Comunes al Hombre y a los Animales. 2. ed. Washington D.C.: Organización Panamericana de la Salud, 1986, p.806-809.

ALENCAR FILHO, R.A. Dioctofimose renal em canino - Observação clínica. O Biológico. São Paulo, v.32 n.2, p.34-36, 1966.

AMATO, J.F.R.; GRISI, L.; ROSA, V.L.M. Reunião dos casos brasileiros de dioctofimose canina, com o registro do caso de mais alta intensidade de infecção por Dioctophyma renale (GOEZE, 1782). Revista Brasileira de Biologia, v.36, p.117-122, 1976.

AUGUSTO FILHO, O.; ARAÚJO, W.N.; PAES, A.C.; MAMPRIM, M.J. Dioctofimíase canina bilateral com vários parasitas - Relato de caso. In: SEMINÁRIO BRASILEIRO DE PARASITOLOGIA VETERINÁRIA, XI. 1999, Salvador, Anais... Salvador: Colégio Brasileiro de Parasitologia Veterinária, 1999, p.175.

BARROS, G. C. Dioctophyme renale (GOEZE, 1782) em cão no Estado do Espírito Santo. Arquivos da Escola de Veterinária da UFMG, Belo Horizonte v.23, p.195-196, 1971. 
BARROS, D.M.; LORINI, M.L.; PERSSON, V.G. Dioctophymosis in the little grison (Galictis cuja) Journal of Wildlife Disease, v.26, n.4, p.538-539, 1990.

BRUN, M.V.; BECK, C.A.C.; MARIANO, M.B.; ANTUNES, R.; PIGATTO, J.A.T. Nefrectomia laparoscópica em cão parasitado por Dioctophyma renale - relato de caso. Arquivo de Ciências Veterinárias e Zoologia da UNIPAR, Umuarama, v.5, n., p.145-152, 2002.

COSTA, H.M.A.; LIMA, W.S. Dioctophyme renale (GOEZE, 1782): ocorrência em Minas Gerais. Arquivo Brasileiro de Medicina Veterinária e Zootecnia, v.40, p.43-245, 1988.

COSTA, H.M.A.; FREITAS, M.G. Alguns helmintos parasitos do guará [Crysocyon brachiurus (Illiger)] com a descrição de Molineus brachiurus .sp. (Nematoda Trichostrongylidae". Arquivos da Escola de Veterinária da UFMG, Belo Horizonte, v.19, p.25-29, 1967.

DACORSO FILHO, P.; LANGENEGGER, J.; DOBEREINER, J. Sobre a infestação e lesões anatomopatológicas produzidas por Dioctophyme renale (GOEZE, 1782) em cães. Veterinária, v.8, p.35-54, 1954.

DUARTE, M.J.F. Helmintos parasitos dos animais domésticos no Estado do Rio de Janeiro. Arquivos da Escola de Veterinária da UFMG, Belo Horizonte, v.33, p.67-98, 1981.

FERNANDES, B.F. Parasitas de animais domésticos do Paraná. Curitiba, 1965. 41 p. Dissertação (Tese de concurso a docência livre da cadeira de Parasitologia) Curso de Medicina Veterinária, Escola de Agronomia e Veterinária da Universidade do Paraná.

GALVÃO, G.R.; PERALTA, A.S.L.; MOREIRA, L.F.M.; COSTA, E.M.F.; SANCHES, O.C.; MATOS, E. Relato de um caso de Dioctofimose em cão da Região Metropolitana de Belém, Estado do Pará. In: SEMINÁRIO BRASILEIRO DE PARASITOLOGIA VETERINÁRIA, XI. 1999, Salvador, Anais... Salvador: Colégio Brasileiro de Parasitologia Veterinária, 1999, p. 174.

GARGILI, A.; FIRAT, I.; TOPARLAK, M.; ÇETINKAYA, H. First case report of Dioctophyme renale (Goeze, 1782) in a dog in Istanbul, Turkey. Turkey Journal Veterinary Animal Science, v.26, p.1189-1191, 2002.

GIOVANNONI, M.; MOLFI, A. O Dioctophyme renale (GOEZE, 1782) no Brasil. Anais da Faculdade de Medicina da Universidade do Paraná, v.3, p.99-144, 1960.

GONÇALVES, P.D.; BARROS, S.S; SIQUEIRA, C.S. Sobre a presença de Dioctophyme renale (Goeze, 1782) no Rio Grande do Sul. (NEMATODA, DIOCTOPHYMOIDEA). Revista da Faculdade de Agronomia e Veterinária. Porto Alegre, v.4, n.1, p.44-94. 1961.
GUTIERREZ, Y.; COHEN, M.; MACHICAO, C.N. Dioctophyme larva in the subcutaneous tissues of a woman in Ohio. American Journal of Surgery and Pathology, Philadelphia, v.13, n.9, p.800-802. 1989.

HANJAN, F.A.; SADIGHIAN, A.; MIKAKHTAR, B.; ARFAA, F. The first report of human infection with Dioctophyma renale in Iran. Transcriptions of Royal Society of Tropical Medicine \& Hygiene, London, v.62, p.647-648, 1968.

IGNJATOVIC, I.; STOJKOVIC, I.; KUTLESIC, C.; TASIC, S. Infestation of the human kidney with Dioctophyma renale. Urologia Internationalis, Basel, v.70, n.1, p.70-73, 2003.

LARA, S. I. M.; TAROUCO, M. R. R.; RIBEIRO, P. B. Helmintos parasitos de Canis familiaris de Pelotas - Rio Grande do Sul. Arquivos da Escola de Veterinária da UFMG, Belo Horizonte, v.33, n.2, p.293-297, 1981.

MARQUES, A.P.; RAFFI, L.L.; RAFFI, M.B.; LOPES, D.C.; SOUZA, R.B.; RAPOSO, J.B. Dioctophyma renale em caninos no município de Pelotas-R.S relato de caso. In: ENCONTRO NACIONAL DE PATOLOGIA VETERINÁRIA, IX. 1999, Belo Horizonte, Resumos... Belo Horizonte: Escola de Veterinária da UFMG, 1999, p. 61.

MAYRINK, K.C.; PAES-DE-ALMEIDA, E.C.; THOMÉ, S.M.G. Dioctophyma renale (GOEZE, 1782) em cães. Caderno Técnico Científico da Escola de Medicina Veterinária da Universidade do Grande Rio, Rio de Janeiro, n.2, p.20-40, 2000.

MIGLIANO, M. F.; MATERA, E. A. Dioctofimose em Canis familiaris, estudo clínico médico e cirúrgico de um caso. Revista de Medicina Veterinária, São Paulo, v.2, n.2, p. 81-93, 1966.

MIRANDA, M. A.; BENIGNO, R. N. M.; GALVÃO, G. R. Dioctophyme renale (GOEZE,1782): localização ectópica e alta intensidade parasitária em Canis familiaris do Pará, Brasil. Arquivo Brasileiro de Medicina Veterinária e Zootecnia, v.44, n.2, p.151153, 1992.

MOLIN, 1860 apud DACORSO FILHO, P.; LANGENEGGER, DOBEREINER, L. Sobre a infestação e lesões Anatomopatológicas produzidas por Dioctophyme renale (GOEZE,1782) em cães. Veterinária, Rio de Janeiro, v.8, n.2, p.35-54, 1954.

MONTEIRO, S.G.; SALLIS, E.S.V.; STAINKI, D.R. Infecção natural por trinta e quatro helmintos da espécie Dioctophyma renale (GOEZE, 1782) em um cão. Revista da Faculdade de Zootecnia, Veterinária e Agronomia PUC-RS, Uruguaiana, v.9, n.1, p.29-32, 2002. 
NEVES, D.S.; MORAIS, A.N. Incidência de Dioctophyme renale na zona urbana de Lages -SC. In: SEMINÁRIO BRASILEIRO DE PARASITOLOGIA VETERINÁRIA, II. 1980, Fortaleza, Anais... Fortaleza: Colégio Brasileiro de Parasitologia Veterinária, 1980, p. 286.

NEVES, D.S.; MORAIS, A.N.; NOGUEIRA, R.H.G.; CHQUILOFF, M.A.G. Ocorrência de Dioctophyme renale (Goeze, 1782) em cães da região de Lages, Estado de Santa Catarina. Arquivo Brasileiro de Medicina Veterinária e Zootecnia, Belo Horizonte, v.35, n.5, p.665-773, 1983.

PACHECO, A.C.; CIRNE, B.R.; CORREA, W.M. Dioctofimíase em cães na região de Botucatu. Archivos do Instituto de Biologia. São Paulo, v.32, n.1 p.1-6, 1965.

PAES-DE-ALMEIDA, E.C.; THOMÉ, S.M.G. Ocorrência da dioctofimose (Dioctophyma renale) canina no município de Silva Jardim - RJ. In: ENCONTRO NACIONAL DE PATOLOGIA VETERINÁRIA, IX. 1999, Belo Horizonte, Resumos... Belo Horizonte: Escola de Veterinária da UFMG, 1999, p. 65.

PROENÇA, M.C. Sobre dois casos de Dioctophyme renale em cão do Rio de Janeiro. Boletim Veterinário do Exército., Rio de Janeiro, v.2, n.3, p. 50-51, 1935.
ROCHA, U.F.R.G.; GRECHI, R. Parasitismo por Dioctophyme renale (GOEZE, 1782) em "preguiça", Choloepus didactylus LINNAEUS, 1785. Revista da Faculdade de Farmácia e Bioquímica. São Paulo, v.3, p.325-334, 1965.

SADIGHIAN, A.; AMINI, F. Dioctophyme renale (GOEZE, 1782) Stiles, 1901 in stray dogs and jackals in Shahsavar Area, Caspian region, Iran. Journal of Parasitology, v.53, p.961, 1967.

SCHIMIDT, F. Contribuição para uma patologia veterinária no Rio Grande do Sul. Egatea, v.9, p.245254, 1924.

SOUZA JÚNIOR, F.L.; PÁDUA, E.B. Dioctophyme renale (GOEZE, 1782) (NEMATODA - DIOCTOPHYMIDAE) em cães de rua da região de Taubaté (São Paulo, Brasil). Revista de Patologia Tropical, v.6, p.7-10, 1977.

TORRES, R.; ALVES, A.; BORGES, A.G.; RAHAL, S.C.; SAKATE, M. Dioctofimíase canina. Ciência Animal, v.11, sup.1, p. 197, 2001.

URANO, Z.; HASEGAWA, H.; KATSUMATA, T.; TORIYAMA, K.; AOKI, Y. Dioctophymatid nematode larva found from human skin with creeping eruption. Journal of Parasitology, Lawrence, v.87, n.2, p.462-465, 2001.

Recebido para publicação: 30/01/2005

Aprovado:

$25 / 04 / 2005$ 